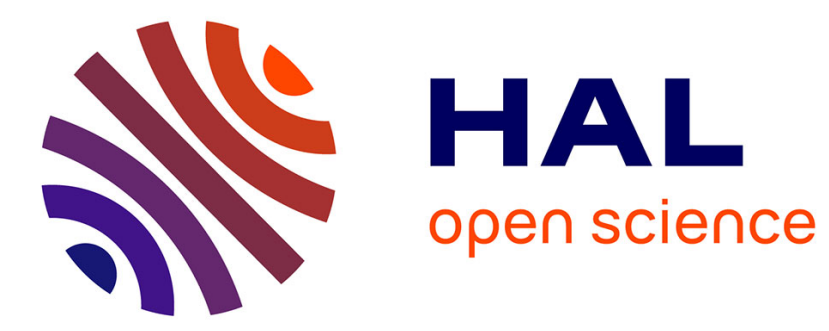

\title{
Évaluation des troubles thymiques par l'étude des données passives : le concept de phénotype digital à l'épreuve de la culture de métier de psychiatre
}

\author{
A. Bourla, F. Ferreri, L. Ogorzelec, C. Guinchard, S. Mouchabac
}

\section{To cite this version:}

A. Bourla, F. Ferreri, L. Ogorzelec, C. Guinchard, S. Mouchabac. Évaluation des troubles thymiques par l'étude des données passives : le concept de phénotype digital à l'épreuve de la culture de métier de psychiatre. L'Encéphale, 2018, 44 (2), pp.168-175. 10.1016/j.encep.2017.07.007 · hal-01960340

\section{HAL Id: hal-01960340 \\ https://hal.sorbonne-universite.fr/hal-01960340}

Submitted on 19 Dec 2018

HAL is a multi-disciplinary open access archive for the deposit and dissemination of scientific research documents, whether they are published or not. The documents may come from teaching and research institutions in France or abroad, or from public or private research centers.
L'archive ouverte pluridisciplinaire HAL, est destinée au dépôt et à la diffusion de documents scientifiques de niveau recherche, publiés ou non, émanant des établissements d'enseignement et de recherche français ou étrangers, des laboratoires publics ou privés. 
Revue de la littérature

\title{
Évaluation des troubles thymiques par l'étude des données passives : le concept de phénotype digital à l'épreuve de la culture de métier de psychiatre
}

\author{
Assessment of mood disorders by passive data gathering: The concept of digital \\ phenotype versus psychiatrist's professional culture
}

\author{
A. Bourla ${ }^{\mathrm{a}, *}$, F. Ferreri $^{\mathrm{a}}$, L. Ogorzelec ${ }^{\mathrm{b}}$, C. Guinchard ${ }^{\mathrm{b}}$, S. Mouchabac $^{\mathrm{a}}$ \\ a UPMC, service de psychiatrie et de psychologie médicale des adultes, hôpital Saint-Antoine, AP-HP, 184, rue du Faubourg-Saint-Antoine, 75012 Paris, \\ France \\ ${ }^{\mathrm{b}}$ LASA-UBFC EA3189, laboratoire de sociologie et d'anthropologie, université Bourgogne Franche-Comté, Besançon, France
}

Mots-clés :

Dépression

Trouble bipolaire

Objet connecté

Culture de métiers

Phénotype digital

\section{R É S U M É}

Objectifs. - La recherche de signes cliniques objectifs est une préoccupation constante des praticiens et des chercheurs en psychiatrie. Le développement récent de certaines technologies (miniaturisation des capteurs, intelligence artificielle) permet d'avoir accès à certaines données dites passives (c'est-à-dire qui ne nécessitent pas d'intervention du patient) jusqu'alors non exploitables et de nouveaux modèles basés sur une sémiologie qui serait médiée par ces nouvelles technologies commencent à se développer avec le concept de phénotype digital : le ralentissement psychomoteur se traduisant par des modifications de l'accéléromètre, la graphorrhée par une augmentation du nombre d'appels et de SMS envoyé, etc. Notre objectif principal est de mettre en évidence le phénotype digital des troubles de l'humeur à l'aide d'une revue sélective de la littérature.

Méthode. - Nous avons conduit une revue sélective de la littérature en interrogeant la base PubMed jusqu'à février 2017 avec les termes [Computer] [Computerised] [Mobile] [Automatic] [Automated] [Machine learning] [Sensor] [Heart rate variability] [HRV] [actigraphy] [actimetry] [digital] [motion] [temperature] [Mood] [Bipolar] [Depression] [Depressive]. Huit cent quarante-neuf articles répertoriés ont été soumis à l'évaluation, 37 articles ont été inclus.

Résultats. - Pour les troubles unipolaires, les smartphones permettent de diagnostiquer la dépression avec une excellente précision en combinant les données du GPS et du journal des appels. Les mesures actigraphiques mettant en évidence une altération diurne dans le fonctionnement basal tandis que les capteurs ECG évaluant la variation de la variabilité du rythme cardiaque (HRV) et la température corporelle semblent être des outils utiles pour diagnostiquer un épisode dépressif. En ce qui concerne les troubles bipolaires, des systèmes qui combinent plusieurs capteurs sont décrits : MONARCA, PRIORI, SIMBA et PSYCHE. Tous ces systèmes associent des données passives et des données actives sur smartphone. À partir d'une synthèse de ces données, un phénotype digital des troubles est proposé en se basant sur l'accéléromètre et le GPS, l'ECG, la température corporelle, l'utilisation du smartphone et l'étude de la voix. Ce phénotype digital vient ainsi remettre en question certains paradigmes cliniques au sein desquels les psychiatres évoluent. L'impact de ces technologies interrogeant profondément la culture de métier du psychiatre.

\footnotetext{
* Auteur correspondant. 
Discussion. - Ces systèmes peuvent être utilisés pour informatiser les caractéristiques cliniques des différents états mentaux étudiés, parfois avec une plus grande précision qu'un clinicien ne pourrait le faire. Par ailleurs, la plupart des auteurs recommandent l'utilisation de données passives préférentiellement aux données actives notamment en cas de troubles bipolaires car les données générées automatiquement réduisent les biais et limitent le sentiment d'intrusion que les autoquestionnaires peuvent causer. L'impact de ces technologies interroge la culture de métier du psychiatre, définie comme une langue spécifique et un ensemble de valeurs communes. Nous abordons les problèmes liés à ces changements en soulignant que l'impact de ce changement de paradigme sur les psychiatres pourrait être important, leur unité semblant remise en cause par des technologies qui modifient profondément la collecte et le traitement des données cliniques.

\section{Keywords:}

Depressive disorder

Bipolar disorder

Connected device

Professional culture

Digital phenotyping

\section{A B S T R A C T}

Objectives. - The search for objective clinical signs is a constant practitioners' and researchers' concern in psychiatry. New technologies (embedded sensors, artificial intelligence) give an easier access to untapped information such as passive data (i.e. that do not require patient intervention). The concept of "digital phenotype" is emerging in psychiatry: a psychomotor alteration translated by accelerometer's modifications contrasting with the usual functioning of the subject, or the graphorrhea of patients presenting a manic episode which is replaced by an increase of SMS sent. Our main objective is to highlight the digital phenotype of mood disorders by means of a selective review of the literature.

Method. - We conducted a selective review of the literature by querying the PubMed database until February 2017 with the terms [Computer] [Computerized] [Machine] [Automatic] [Automated] [Heart rate variability] [HRV] [actigraphy] [actimetry] [digital] [motion] [temperature] [Mood][Bipolar] [Depression] [Depressive]. Eight hundred and forty-nine articles were submitted for evaluation, 37 articles were included.

Results. - For unipolar disorders, smartphones can diagnose depression with excellent accuracy by combining GPS and call log data. Actigraphic measurements showing daytime alteration in basal function while ECG sensors assessing variation in heart rate variability (HRV) and body temperature appear to be useful tools to diagnose a depressive episode. For bipolar disorders, systems which combine several sensors are described: MONARCA, PRIORI, SIMBA and PSYCHE. All these systems combine passive and active data on smartphones. From a synthesis of these data, a digital phenotype of the disorders is proposed based on the accelerometer and the GPS, the ECG, the body temperature, the use of the smartphone and the voice. This digital phenotype thus brings into question certain clinical paradigms in which psychiatrists evolve. Conclusion. - All these systems can be used to computerize the clinical characteristics of the various mental states studied, sometimes with greater precision than a clinician could do. Most authors recommend the use of passive data rather than active data in the context of bipolar disorders because automatically generated data reduce biases and limit the feeling of intrusion that self-questionnaires may cause. The impact of these technologies questions the psychiatrist's professional culture, defined as a specific language and a set of common values. We address issues related to these changes. Impact on psychiatrists could be important because their unity seems to be questioned due to technologies that profoundly modify the collect and process of clinical data.

\section{Introduction}

Le diagnostic des troubles thymiques est actuellement purement clinique et repose sur la mise en évidence de symptômes qui peuvent être soit subjectifs (tristesse, anhédonie, etc.), soit potentiellement objectivables et visibles par les tiers (ralentissement psychomoteur, troubles attentionnels, troubles instinctuels, etc.). Dans ce contexte, la recherche de biomarqueurs est l'un des enjeux majeurs de la recherche dans ce domaine.

La notion de " signature digitale " d'une pathologie ou " phénotype digital "[1] - aussi appelé par certains biomarqueur électronique [2] - développée notamment par John Torous fait référence à la " captation " par des outils de mesures informatisés de certaines caractéristiques propres à un trouble psychiatrique. Certains comportements ou symptômes seraient " quantifiables ", et cette sémiologie médiée par l'outil informatique constituerait une "e-sémiologie ": par exemple une altération de la symptomatologie motrice traduite par des modifications de l'accéléromètre qui trancherait avec le fonctionnement habituel du sujet, ou la graphorrhée des patients présentant un épisode maniaque qui se voit remplacée par une augmentation du nombre d'envoi des SMS.
Des modèles basés sur ces nouveaux "signes sémiologiques " commencent à émerger notamment en s'appuyant sur des technologies innovantes. Pour mettre en évidence les symptômes dits " objectifs » de la dépression ou de la bipolarité [3], les critères le plus souvent étudiés sont ceux qui concernent les aspects moteurs (ralentissement et agitation), les troubles du sommeil (insomnie ou hypersomnie) ainsi que des données biométriques détectables par des capteurs (fréquence cardiaque, température, etc.). La miniaturisation des capteurs et l'usage ubiquitaire des smartphones permettant ainsi de collecter un nombre de données important auxquelles les psychiatres n'avaient jusqu'alors pas accès. Cette méthode de collecte fait appelle à la gestion des données dites passives : aucune intervention n'est nécessaire et le recueil en " tâche de fond " s'effectue parfois sans même que le sujet ne s'en rende compte, réduisant le poids de l'observateur au minimum. Ce terme s'oppose à celui de données actives, qui nécessitent l'implication du patient dans le recueil comme c'est par exemple le cas pour l'évaluation écologique momentanée (EMA) pour laquelle le patient renseigne des données directement [4] en répondant à des questions.

Toutes ces évolutions pourraient venir bouleverser les pratiques et surtout les praticiens dans leurs croyances, leur éthique 
et leurs représentations puisqu'il s'agit là d'un véritable changement de paradigme qui vient interroger la culture de métier de psychiatre.

Notre objectif est donc de mettre en évidence le phénotype digital des troubles de l'humeur : dépression unipolaire, dépression bipolaire et manie, à l'aide des principales données passives retrouvées dans la littérature.

\section{Méthode}

Nous avons ainsi mené une revue sélective de la littérature en interrogeant la base PubMed jusqu'à février 2017 sur les titres avec les termes [Computer] OR [Computerised] OR [Mobile] OR [Automatic] OR [Automated] OR [Machine learning] OR [Sensor] OR [Heart rate variability] OR [HRV] OR [actigraphy] OR [actimetry] OR [digital] OR [motion] OR [temperature] avec chacun des termes (AND) suivants : [Mood] $(n=90)$, [Bipolar] $(n=176)$, [Depression] $(n=457)$, [Depressive] $(n=108)$, [Manic] $(n=11)$, [Mania] $(n=7)$ (Fig. 1). Huit cent quarante-neuf articles ont été soumis à évaluation avec les critères d'inclusion suivants : article en langue française ou anglaise, traitant de l'évaluation de la dépression ou de la manie à l'aide de capteurs. Étaient exclus les articles soit sans rapport avec le sujet, soit redondants, soit en rapport avec l'EMA, l'imagerie ou avec des technologies utilisées à visée thérapeutique ou de psycho-éducation. Les abstracts de 523 articles ont été analysés et 37 articles ont été inclus dans cette revue.

Pour présenter les données nous avons décidé de les catégoriser en fonction d'abord du type de pathologie (trouble unipolaire et bipolaire) et à chaque fois en fonction du capteur principal (données téléphoniques, électrocardiogramme [ECG], capteur de localisation [GPS], etc.).

\section{Résultats}

\subsection{Troubles dépressifs unipolaires}

Plusieurs types de capteurs ont été utilisés dans des études portant spécifiquement sur les troubles dépressifs unipolaires. Certaines études utilisent plusieurs capteurs en même temps, c'est notamment le cas pour celles qui utilisent les smartphones. D'autres études se concentrent spécifiquement sur un type de capteur.

\subsection{1. Évaluation par capteurs multiples}

L'utilisation des capteurs présents en série sur de nombreux smartphones permet de mettre en évidence certains symptômes du retentissement de la maladie. Il est ainsi montré [5] que l'association des données issue du GPS, du détecteur de luminosité, de l'accéléromètre et du journal d'appel permet de détecter des modifications dans les interactions sociales spécifiquement lorsque les patients sont déprimés à l'aide de l'application « Mobilyze! ». On retrouve aussi une excellente corrélation entre les données issues des capteurs (GPS et données téléphoniques) et l'échelle de dépression PHQ-9 [6] : 40 patients adultes ont bénéficié d'une double évaluation, d'abord par une échelle PHQ-9 puis par smartphone durant deux semaines à l'aide d'une application appelée " Purple Robot » qui permettait d'analyser le GPS du téléphone et d'accéder aux données concernant l'utilisation du téléphone. Quatre données, collectées à partir de deux sources (le GPS du téléphone et l'utilisation des données téléphoniques), permettent de classer avec une précision de 86,5\% les patients en deux groupes (PHQ$9>5$ et $\mathrm{PHQ}<5$ ). Les auteurs de cette étude soulignent par ailleurs la supériorité de données issues du GPS sur celles obtenues par EMA [7]. Plus récemment, l'application MOSS [8] a permis de détecter la dépression grâce à un algorithme d'apprentissage automatique

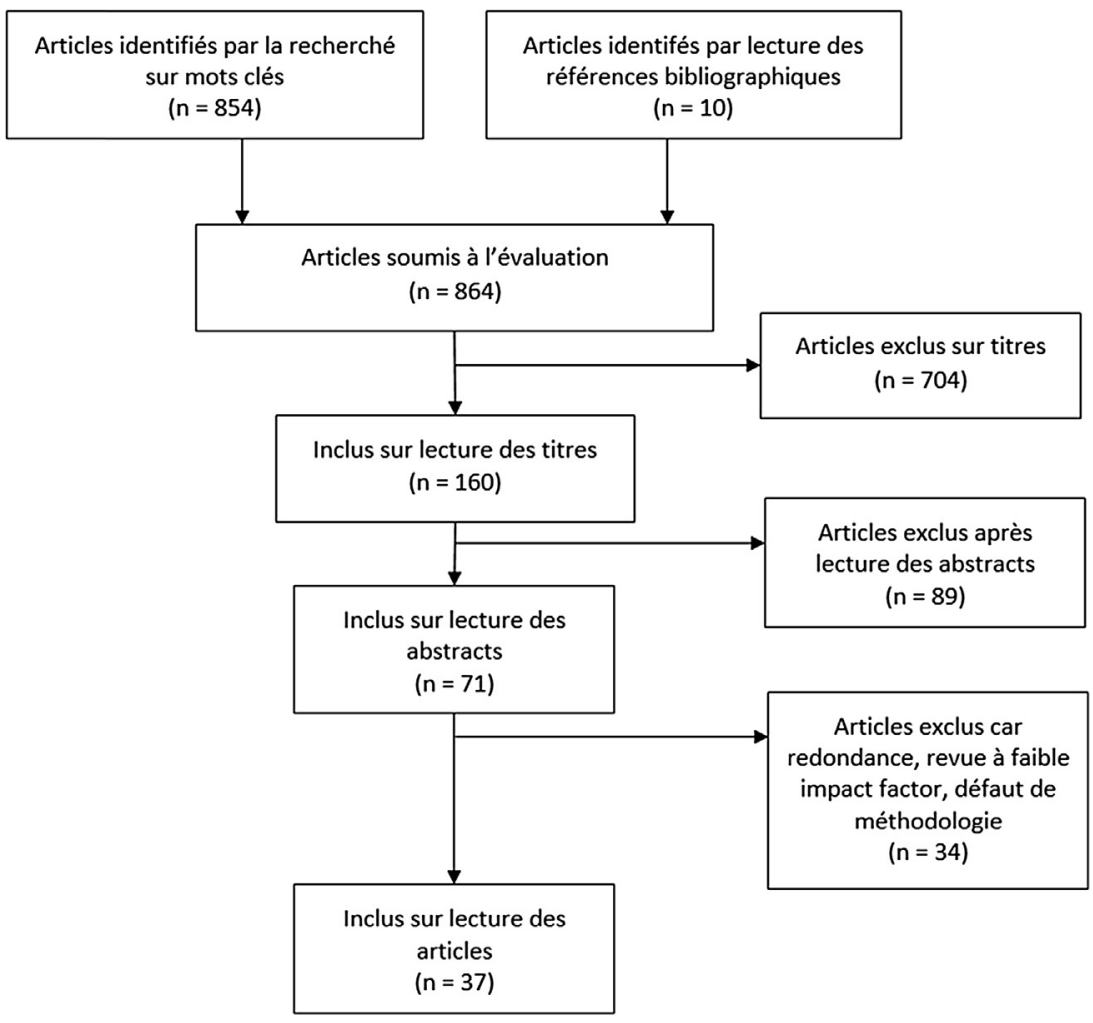

Fig. 1. Diagramme de flux. 
utilisant des données issues du GPS, du wifi, de l'accéléromètre et de l'utilisation du téléphone avec une précision de $60 \%$.

\subsection{2. Évaluation par capteurs de mouvement}

Les capteurs de mouvement sont de plusieurs types (actigraphes, accéléromètres associés à des gyroscopes, podomètres) et peuvent être placés soit sur les poignets (montre et bracelets connectés ou non), soit sur le thorax (bande thoracique ou vêtement équipé de capteur), soit dans un smartphone. Une récente revue de la littérature, menée par Burton et al. en 2013 [9], résume les études portant sur le monitorage moteur de la dépression : 19 articles (soit 16 études, 412 patients) ont été inclus, les évaluations duraient entre 2 et 30 jours et portaient sur des données diurnes (15 articles) et/ou nocturnes (14 articles). Il apparaît que le seul paramètre moteur qui est significativement altéré chez les sujets déprimés, par rapport à des sujets témoins non déprimés, est celui portant sur les " activités diurnes ". Ce paramètre s'améliore chez les patients en cas de traitement de la dépression par antidépresseurs. Cette même étude retrouve aussi des différences en ce qui concerne l'analyse de la marche (diminuée chez les sujets déprimés).

\subsection{3. Évaluation par capteurs ECG}

La variabilité de fréquence cardiaque (VFC) [10] est définie comme le degré de fluctuation de l'intervalle entre deux contractions cardiaques. Elle est dépendante du système nerveux autonome et de la balance sympathique/parasympathique, ainsi que d'autres facteurs (respiration, température, variations diurnes, métabolisme). Cette mesure est utilisée comme indicateur d'une dérégulation cardiaque et est associée à un risque majoré de morbimortalité après un accident cardiovasculaire, et de manière plus globale à un état de santé altéré [11]. Les liens entre dépression et VFC ont été rappelés dans une revue de la littérature réalisée en 2010 par Kemp [12] et plus récemment par Jangpangi et al. [13]. Kemp retrouve 18 articles comparant 673 patients déprimés à 407 sujets témoins et met en évidence l'existence d'une diminution de la VFC chez les patients déprimés avec un effet sévérité-dépendant. Cette diminution est importante puisqu'une étude [14] comparant la VFC de patients déprimés, de sujets sains et de patients transplantés cardiaques (représentant le « paradigme du cœur dénervé ") retrouve des VFC comparables entre les groupes transplantés et déprimés. L'étude de la VFC grâce à un capteur ECG permet ainsi de discriminer les sujets sains des sujets déprimés. De plus l'étude de la VFC pourrait permettre de discriminer différents types de dépression : une étude publiée en 2015 [15] compare la VFC de trois groupes de patients (591 patients présentant une dépression unipolaire, 116 patients présentant une dépression bipolaire dans un contexte de trouble bipolaire de type 2 et 421 sujets témoins) et confirme qu'il existe un lien entre la sévérité de la maladie dépressive (quel que soit le groupe) et la diminution de la VFC. Les patients unipolaires se distinguent des bipolaires par un meilleur profile cardiologique : les patients bipolaires présentent une fréquence cardiaque plus élevée, une VFC plus basse et donc probablement un tonus parasympathique diminué (et un tonus sympathique augmenté) comparativement aux patients unipolaires. Il existe plusieurs limites méthodologiques à ces études : d'une part, les groupes de patients déprimés étaient en général sans comorbidités somatiques (notamment cardiaque puisque les maladies cardiaques influencent la VFC), d'autre part, certaines études limitent l'impact des traitements alors qu'il existerait un effet des traitements antidépresseurs [16,17] sur la VFC (tricycliques et probablement ISRS notamment Paroxetine).

\subsection{4. Évaluation par capteurs de température corporelle}

De même que la VFC, la température corporelle est soumise à des régulations faisant intervenir plusieurs systèmes (neurohormones, hypothalamus, système nerveux autonome) et par conséquent les altérations du tonus sympathique et parasympathique induites par la dépression peuvent aussi se traduire par ce biais. Plusieurs études rapportent une corrélation négative entre la température (prise avec un capteur au niveau d'un doigt) et la dépression [18,19], sauf en ce qui concerne la température nocturne qui serait plus élevée chez les sujets déprimés [20]. Par ailleurs les variations (amplitudes) de température seraient aussi amoindries [21] et il y aurait une avance de phase de 2 à 4 heures [22] chez les sujets déprimés.

\subsection{Troubles bipolaires}

L'étude des données passives dans le trouble bipolaire est beaucoup plus développée que dans le trouble dépressif unipolaire car la symptomatologie maniaque se prête particulièrement bien à ce type de collecte. De plus, vu les difficultés relatives au recueil des données actives chez ces patients lors des phases prodromales ou lorsque la symptomatologie devient sévère, il était logique que des techniques ne faisant pas appel à la participation du patient se développent. Trois types de données passives sont particulièrement intéressants : celles relatives à la parole, celles en lien avec la participation aux activités sociales [23] et celles correspondant aux données biométriques.

\subsection{1. Évaluation par smartphone}

Développé par l'équipe de Maria Faurholt-Jepsen et financé par un programme de recherche de l'Union européenne, le FP7 ("Seventh Framework Programme »), le système MONARCA (pour MONitoring, treAtment and pRediCtion of bipolAr disorder episodes) est une application pour smartphone Android qui a été testée dans plusieurs études contrôlées-randomisées [24,25]. Cette application permet à la fois d'analyser des données actives et des données passives. L'acquisition des données actives se fait par questionnaires avec auto-évaluation quotidienne de l'humeur (cotée de +3 à -3 ), du sommeil (en nombre d'heures), du traitement, des activités et d'un certain nombre de symptômes pour lesquels le patient doit dire s'ils sont présents ou absents (irritabilité, éléments de mixité, troubles cognitifs, prise d'alcool, stress).

Les données passives sont issues de l'analyse de l'utilisation du téléphone en émission et en réception pour trois facteurs : le nombre d'appels, la durée des appels et le nombre de messages. Le nombre et la durée des appels permettant de renseigner sur la parole, tandis que le nombre d'appels et le nombre de messages permettant de renseigner sur l'investissement dans les activités sociales. Enfin il existe une fonction (appelée " feedback loop ») permettant au psychiatre traitant d'être informé de l'état thymique du patient afin de pouvoir intervenir si besoin. L'étude MONARCA [2] a permis d'inclure 61 patients randomisés en deux groupes : un groupe $(n=30)$ avec l'étude des données actives et passives et un groupe $(n=31)$ avec l'étude des données passives uniquement, permettant de créer un groupe commun $(n=61)$ où les données passives de tous les participants étaient analysées. Il ressort des résultats que seules les données passives permettent de classifier correctement les patients en différents groupes de sévérité (asymptomatique, modéré, sévère) corrélés aux échelles HDRS et YMRS. Ainsi, la durée des appels est par exemple corrélée à la fois aux épisodes dépressifs et aux épisodes maniaques, ce qui pourrait s'expliquer par le fait que les patients déprimés parlent plus lentement tandis que les patients en phase maniaque parlent plus longtemps. Une seconde étude, MONARCA II, est en cours de développement afin de déterminer si l'utilisation du système peut avoir une influence sur le cours de la maladie.

Une autre équipe a utilisé le système MONARCA [26] sur un faible nombre de patients $(n=5)$, mais en associant à l'application un système autonome qui élargit la collecte des données passives avec notamment des données audio (via le microphone 
du smartphone) et des données motrices (via l'accéléromètre et le GPS). Concernant les données motrices, les résultats sont concordants avec ceux des autres études avec notamment une augmentation de l'activité associée aux symptômes maniaques et une diminution associée aux symptômes dépressifs. Concernant les données audio, elles ont été extraites à l'aide des logiciels OpenEar et Praat qui avaient déjà été utilisés précédemment avec succès pour classer les émotions [27]. Leur étude a permis de mettre en évidence un temps de latence des réponses augmenté chez les patients déprimés, une modification de la durée des appels, un nombre de pauses et une durée des pauses augmentés. L'un des patients de l'étude a présenté une alternance de différentes phases au cours de l'étude, passant de l'euthymie à la manie puis à la dépression. L'analyse de ce cas permet de mettre en évidence que le nombre de pauses diminuait significativement lors de la phase maniaque et augmentait lors de la phase dépressive. Une autre étude [28] soutenue par le projet MONARCA a montré qu'il était possible de classifier un état mental (dépression ou manie) avec une précision de $97 \%$ en utilisant plusieurs capteurs (GPS, accéléromètre, son, utilisation du téléphone).

\subsection{2. Évaluation par analyse de la voix}

Le système PRIORI est une application pour smartphone destinée à détecter les symptômes de la maladie bipolaire de manière précoce afin de déterminer l'état thymique de l'utilisateur par l'analyse de la voix. Développé conjointement par une équipe d'ingénieurs et une équipe de psychiatres au sein du département CSE (Computer Science and Engineering) de l'université du Michigan. Une étude pilote sur six patients présentant un trouble bipolaire de type 1 à cycle rapide évalués durant 12 mois a été réalisée [29] : le système analyse la voix et les silences à la fois lors des appels habituels du patient et lors d'appels hebdomadaires programmés et structurés. Parallèlement, le patient est évalué de manière hebdomadaire par un psychiatre et c'est la concordance des deux évaluations qui permet d'établir un profil spécifique pour chaque patient. Le logiciel possède un module d'entraînement qui lui permet de s'améliorer avec le temps en détectant des variations de plus en plus subtiles. Là encore, une fonction " feedback loop » est intégrée pour permettre au clinicien d'être alerté si nécessaire en cas de modification thymique. Après extraction des données, une méthode d'apprentissage automatique (SVM) a été utilisée et les résultats permettent de différencier correctement l'euthymie de l'hypomanie avec une aire sous la courbe (AUC) de 0,81 (l'AUC fournit un index simple et quantitatif de la performance d'un test) et de la différencier de la dépression avec un AUC de 0,67. Une seconde étude [30] de plus grande ampleur (sur 50 patients) est en cours en vue de valider les résultats de l'étude pilote.

\subsection{3. Évaluation par capteur ECG}

Le système PSYCHE (Personalised monitoring SYstems for Care in mental HEalth) est un projet financé lui aussi par le programme de recherche de l'Union européenne FP7. Développé conjointement par plusieurs sociétés et universités européennes et fabriqué par une société privée, ce système repose sur une architecture qui associe l'utilisation de données passives mesurées par un tee-shirt possédant plusieurs capteurs et la mesure de données actives par smartphone [31]. Les données passives collectées concernent l'ECG (mesure de l'intervalle RR avec calcul de la VFC) et l'activité respiratoire (mesure de la fréquence respiratoire et calcul d'un certain nombre de caractéristiques type maximum, minimum, amplitude, variation, etc.). Le choix de l'ECG pour caractériser un épisode thymique repose sur plusieurs études montrant des différences de VFC chez les patients bipolaires par rapport aux sujets témoins avec notamment des caractéristiques différentes pour les épisodes hypomanes ou mixtes [32], et d'après certains auteurs la VFC permettrait de classer correctement les patients dans l'un des quatre états thymiques (euthymique, hypomane, déprimé ou mixte) avec une précision de $99 \%$ [33]. Ainsi, la VFC est diminuée [34] comparativement aux sujets sains lors des phases dépressives, y compris dans des phases prodromales [35], et augmentée lors des phases maniaques $[36,37]$. Lors des phases d'euthymie, la VFC serait plus basse que chez les témoins sains [38].

Dans une étude préliminaire de validation [39], trois patients bipolaires (type 1 ou 2) avaient été inclus et une évaluation thymique avait été réalisée à l'aide de plusieurs échelles (IMS, POMS, QIDS, YMRS) afin de classer le patient dans une catégorie de trouble thymique (euthymie, dépression sévère ou modérée, état mixte dépressif ou maniaque, état mixte associé à une dépression sévère ou à une manie sévère, manie sévère, hypomanie). Les sujets ont porté le prototype sur une période de 90 jours avec aux moins six évaluations cliniques sur la période. L'acquisition des données s'étalait sur 16 heures lorsque le tee-shirt était porté. Plusieurs techniques d'apprentissage automatiques ont été utilisées avec une précision de classification pouvant aller jusqu'à 97,96\%. Les auteurs affirment ainsi que deux variables issues du système cardiorespiratoire suffisent pour affirmer avec une bonne précision dans quel état thymique se trouve un patient bipolaire. Le faible nombre de sujets $(n=3)$ et l'acceptabilité de vêtements connectés sont des limites importantes. Concernant le tee-shirt connecté, il existe certaines réserves puisque sur une étude d'acceptabilité du tee-shirt portant sur cinq patients, tous avaient affirmé qu'ils refuseraient de le porter pour une période trop longue d'autant qu'à l'usage certains le trouvaient " trop serré " ou " trop chaud ».

Une équipe a proposé d'intégrer une analyse de la voix sur la plateforme PSYCHE en se basant sur les mêmes données que pour les systèmes PRIORI et MONARCA : dans cette étude [40], l'analyse des enregistrements de la voix de six patients bipolaires dans deux états thymiques différents ( 3 patients hypomanes puis euthymiques et 3 patients déprimés puis euthymiques) lors d'une tâche de lecture et lors d'une tâche d'expression libre avec un algorithme basé sur une segmentation automatique de la voix, a permis de retrouver une différence significative entre les deux états.

\subsection{4. Évaluation par actigraphie}

Une équipe allemande a réalisé une étude pilote [41] à l'aide d'un système appelé SIMBA pour Social Information Monitoring for Patients with Bipolar Affective Disorder. Là encore il s'agit d'un système de recueil de données actives (des questionnaires apparaissent de manière régulière sur l'écran) associé à un recueil de données passives avec notamment le GPS permettant une mesure de distance, l'accéléromètre, la connexion aux antennes relais, le nombre et la durée des appels émis, le nombre de SMS envoyés par jour.

Toutes ces données ont été collectées chez 13 patients bipolaires et des évaluations cliniques de leur état thymique étaient réalisées régulièrement avec des échelles HDRS et YMRS. Les résultats montrent qu'il existe une bonne corrélation entre une humeur dépressive mesurée cliniquement, données actives (déclaration d'une humeur dépressive), les données sociales (diminution des SMS émis) et données biométriques (diminution de l'activité physique mesurée par la connexion aux antennes). En ce qui concerne le versant maniaque, il existe une bonne corrélation avec les données sociales (augmentation de la communication) et les données biométriques (augmentation ou diminution de l'activité physique mesurée par la distance GPS). Une autre étude [42] retrouve un profil d'activité différent entre les jours où la thymie est considérée comme dépressive et les jours d'euthymie.

Concernant l'utilisation de l'actigraphie nocturne, une étude [43] montre qu'il est possible de différencier un patient bipolaire en rémission d'un patient témoin avec $89 \%$ de précision à l'aide de trois variables actigraphiques (durée moyenne de sommeil, latence d'endormissement, variabilité sur 21 jours) et une variable clinique 
Tableau 1

Phénotype digital des troubles thymiques.

\begin{tabular}{|c|c|c|c|}
\hline & Dépression unipolaire & Dépression bipolaire & Phase maniaque \\
\hline $\begin{array}{l}\text { Accéléromètre } \\
\text { GPS }\end{array}$ & $\begin{array}{l}\text { Diminution des activités diurnes } \\
\text { Diminution de la marche [9] }\end{array}$ & $\begin{array}{l}\text { Niveau d'activité plus faible ; début plus tardif } \\
\text { des activités ; pic d'activité à midi ; faible } \\
\text { activités en soirées [41] }\end{array}$ & $\begin{array}{l}\text { Augmentation des activités et des localisations } \\
\text { [41] }\end{array}$ \\
\hline ECG & $\begin{array}{l}\text { Diminution sévérité-dépendante de la } \\
\text { VFC ; augmentation de la VFC basse } \\
\text { fréquence et du ratio basse } \\
\text { fréquence/haute fréquence ; } \\
\text { diminution de la VFC haute fréquence } \\
\text { [12-15] }\end{array}$ & $\begin{array}{l}\text { Fréquence cardiaque plus élevée et VFC plus } \\
\text { basse que chez les unipolaires [32-34] } \\
\text { Chez bipolaire type } 2 \text { : augmentation du ratio } \\
\text { basse fréquence/haute fréquence et } \\
\text { diminution de la VFC haute fréquence [15] }\end{array}$ & $\begin{array}{l}\text { Diminution de l'intervalle RR (augmentation } \\
\text { de la fréquence cardiaque), de la variance, de la } \\
\text { VFC basse fréquence, et de la VFC haute } \\
\text { fréquence ; augmentation du ratio basse } \\
\text { fréquence/haute fréquence [37] ; } \\
\text { augmentation de la VFC [36] }\end{array}$ \\
\hline Température & $\begin{array}{l}\text { Diminution de la température }[18,19] \\
\text { Augmentation de la température } \\
\text { nocturne [20] } \\
\text { Amplitude diminuée [21] } \\
\text { Avance de phase [22] }\end{array}$ & Absence de donnée & Absence de donnée \\
\hline $\begin{array}{l}\text { Smartphone } \\
{[24,25]}\end{array}$ & $\begin{array}{l}\text { Diminution de l'utilisation du } \\
\text { smartphone (nombre de SMS, nombre } \\
\text { d'appels) }\end{array}$ & Modification de la durée des appels & $\begin{array}{l}\text { Nombre d'appels augmenté ; durée des appels } \\
\text { augmentée ; nombre de messages augmenté }\end{array}$ \\
\hline Voix $[29,30]$ & Absence de donnée & $\begin{array}{l}\text { Temps de latence des réponses augmenté ; } \\
\text { nombre de pauses et durée des pauses } \\
\text { augmentés }\end{array}$ & Nombre de pauses diminué \\
\hline
\end{tabular}

VFC : variabilité de fréquence cardiaque.

(une sous-échelle de la PSQI). Enfin, on retrouverait aussi une augmentation des mouvements durant le sommeil (notamment entre 2 heures et 7 heures du matin) et une amplitude du rythme circadien diminué [44].

\subsection{Le phénotype digital}

À partir de données issues de ces études, le Tableau 1 résume les éléments composant le phénotype digital des troubles thymiques.

Par ailleurs, les patients bipolaires euthymiques ou unipolaires en rémission semblent aussi avoir des phénotypes digitaux individualisables, notamment par étude de la VFC et de l'actigraphie nocturne $[35,38,43,44]$.

\section{Discussion}

Tous ces systèmes permettent de traduire informatiquement des caractéristiques cliniques propres aux divers états mentaux étudiés, avec parfois une meilleure précision que ce que pourrait faire un clinicien, et la possibilité de le faire à distance. La plupart des auteurs préconisent l'emploi des données passives préférentiellement aux données actives dans le cadre des troubles bipolaires car ce type de données générées automatiquement permet de limiter les biais et de limiter le sentiment d'intrusion que peuvent occasionner les autoquestionnaires (surtout s'ils doivent être remplis régulièrement ou s'ils apparaissent en " pop-up »). Ces études mettent en avant la bonne acceptabilité et la bonne compliance des patients puisqu'aucun ne se serait senti " observé " durant ces études, et il semblerait que l'utilisation d'un smartphone soit moins stigmatisante que l'utilisation d'un dispositif spécifique (ex. : bracelet connecté).

Ces évolutions appellent un changement des modes de pensée ainsi que des manières de faire, elles interrogent ce qu'on pourrait désigner comme la " culture de métier » des psychiatres. Cette notion, issue de la sociologie des professions, désigne le fait que, dans leurs activités, les professionnels ne se réfèrent pas seulement à un ensemble plus ou moins lié de connaissances théoriques et de recettes techniques apprises en formation initiale ou même accumulées avec l'expérience. Ce qu'il faut prendre en compte, c'est la façon dont un métier cimente ces différentes connaissances qui, sans cela, resteraient éparses et inefficaces. De ce point de vue, une culture de métier forme une totalité de dispositions réunies par la socialisation des individus dans un groupe possédant non seulement des conditions de travail similaires, mais surtout partageant un langage spécifique et un ensemble de valeurs communes. Les liens entre les divers savoirs et savoir-faire que tisse une culture de métier sont d'autant plus efficaces qu'ils sont moins réfléchis et paraissent aller de soi. Il y a là une sorte de principe d'économie qui libère celui qui agit et le laisse disponible pour certaines tâches nécessitant une grande attention.

On retiendra surtout qu'une telle culture est susceptible de coder identiquement certaines expériences qui deviennent alors communes et qu'elle est également capable de susciter un certain nombre de comportements spécifiques qui paraissent dès lors aller de soi. Ces considérations sont particulièrement valables dans le monde médical qui revendique une culture identitaire forte (corporation, ordre, spécialité, rites de passage de type concours) et dont les manifestations visibles sont reconnues de manière tacite, comme partagées par les gens du métier (connaissance de l'examen physique, des techniques d'investigation, du raisonnement anatomoclinique, etc.) et que seuls eux sont habilités à pratiquer. Tout médecin sait comment s'adresser à un patient, comment il peut communiquer à propos de ce patient avec ses confrères, en parler avec un infirmier ou un travailleur social.

La culture du métier de psychiatre se distingue de celle des autres spécialités médicales par la prédominance de la clinique dans le raisonnement [45], du fait de l'absence d'examens complémentaires reconnus et validés, mais aussi en lien avec la place importante faite à l'intuition, au contre-transfert et au sens clinique. De même, la dimension psychothérapeutique qui consiste à " être " thérapeute et à aider le patient avec sa propre personne met le psychiatre à part. Ces caractéristiques de la culture de métier des psychiatres facilitent les relations entre ceux qui les possèdent. Elles renforcent le sentiment d'appartenance à un même groupe parce que ceux qui sont ainsi socialisés construisent de la même manière la signification de leurs expériences et n'ont pas besoin d'expliciter ce qui, pour eux, " va de soi ". Partant de là, on comprendra que la culture de métier soit un moyen de se positionner par rapport aux autres professionnels - qu'ils pratiquent le même métier ou un autre. On peut même dire ici que la culture de métier trace une frontière nette entre ceux qui la possèdent et les autres. Ces derniers étant, dès lors, impitoyablement rejetés en dehors du cercle des " membres".

Cependant, dans la littérature recensée, cette unité paraît mise en cause, retraçant cette frontière à l'intérieur même de la profession, du fait de l'émergence des techniques qui modifient 
profondément le recueil et le traitement des données cliniques. En allant interroger la prévalence de l'approche psychodynamique et en lui opposant une vision plus organique de la psychiatrie, plusieurs questions peuvent ainsi se poser : Les psychiatres craignent-ils de se faire remplacer par ces machines ? Quelles aides technologiques sont-ils prêts à solliciter et pour quelles tâches? Peuvent-ils supporter la comparaison avec certaines technologies qui semblent faire preuve d'une plus grande précision prédictive ? Au contraire, leur rôle de médecin peut-il être mieux affirmé grâce à ces dispositifs ?

Dans un travail mené sur le sujet [46], portant sur 515 psychiatres et dont l'objectif consistait à interroger l'acceptabilité de ces nouvelles technologies (définie selon les critères classiques : intérêt, utilisabilité, fiabilité, etc.) à l'aide d'une méthode basée sur des scénarios (évaluant la prédiction par l'imagerie médicale, le recueil de données passive et active), il a été mis en évidence qu'il existait plusieurs types de profils de psychiatres possédant chacun une assez forte cohérence interne. Ainsi, un premier type (20\% des psychiatres), défini sociologiquement comme " scientifique " (utilisation en pratique des données biométriques issues d'examens complémentaires ou de l'examen physique, utilisation d'algorithmes), significativement associé avec le sexe masculin, lié à une pratique en milieu universitaire (CCA, MCU, PU-PH) ainsi qu'à une pratique orientée neurobiologique correspondait à un haut degré d'acceptabilité. À l'inverse, un autre type (25\% des psychiatres) pouvait être qualifié de " psychologique ", significativement associée avec le sexe féminin, lié à une pratique pédopsychiatrique ou de psychiatrie légale, à une pratique libérale et à une orientation psychanalytique, était associé à un refus majeur de ces dispositifs, soulignant certains risques (perte du lien thérapeutique, risque de faux positif ou faux négatif, focalisation excessive entraînant le risque de majorer le stress du patient, intrusion d'une dimension prédictive ou du moins d'une détection très précoce des troubles) [46]. Un type intermédiaire (55\% des psychiatres), composé des $\mathrm{PH}$ et assistants, avec une pratique en psychiatrie adulte, et une orientation déclarée de type " intégrative ", était associé à une acceptabilité " potentielle " de ces dispositifs, c'est-à-dire conditionnée en fonction du type de patient, de la situation, de la pathologie. Cette approche interdisciplinaire, associant sociologie et psychiatrie, menée en termes de " culture de métier ", a permis de confirmer, mais surtout de nuancer, les résultats récemment obtenus par $\mathrm{C}$. Borelle dans le domaine de la pédopsychiatrie à partir d'une lecture en matière d'« autorité » [47]. Elle a mis en évidence, à propos de l'acceptabilité de technologies engageant des pratiques concrètes, un ensemble de tensions qui traduisent une modification profonde de la culture de métier des psychiatres.

\section{Conclusion}

Il apparaît en fin de compte que la notion de phénotype digital de la pathologie est la traduction informatique des signes objectivables de la maladie psychique, et devrait donc être entendue comme un moyen de renforcer les capacités d'observation des psychiatres. Concernant le trouble dépressif, les principaux éléments sont la diminution des paramètres fonctionnels et biologiques (diminution des activités et de la marche, diminution du nombre d'appels et de SMS, diminution de la température et de la VFC) tandis que la phase maniaque retrouve le phénomène inverse (augmentation des activités, du nombre d'appels et de la VFC) tel que l'on peut s'y attendre.

D'importantes considérations éthiques et déontologiques entravent l'acceptation de ces technologies qui semblent très fortement conditionnées par le degré de "scientificité " ou de " médicalisation " des psychiatres. Pour être utilisés, ces outils doivent donc être expliqués et adaptés aux différents profils tout en prenant en compte les risques inhérents à leur utilisation (captation des données, risque de faux positif, etc.). La participation des patients, aidants et autres professionnels de santé s'avère indispensable à la conception et l'évaluation de ces nouveaux outils.

\section{Déclaration de liens d'intérêts}

Les auteurs déclarent ne pas avoir de liens d'intérêts.

\section{Références}

[1] Torous J, Kiang MV, Lorme J, et al. New tools for new research in psychiatry: a scalable and customizable platform to empower data driven smartphone research. JMIR Ment Health 2016;3(2):e16.

[2] Faurholt-Jepsen M, Vinberg M, Frost M, et al. Smartphone data as an electronic biomarker of illness activity in bipolar disorder. Bipolar Disord 2015;17(7):715-28.

[3] Prociow PA, Crowe JA. Development of mobile psychiatry for bipolar disorder patients. Conf Proc IEEE Eng Med Biol Soc 2010;2010:5484-7.

[4] Schwartz JE, Stone AA. Strategies for analyzing ecological momentary assessment data. Health Psychol 1998;17(1):6-16.

[5] Burns MN, Begale M, Duffecy J, et al. Harnessing context sensing to develop a mobile intervention for depression. J Med Internet Res 2011:13(3):e55.

[6] Saeb S, Zhang M, Karr CJ, et al. Mobile phone sensor correlates of depressive symptom severity in daily-life behavior: an exploratory study. J Med Internet Res 2015;17:e175.

[7] Saeb S, Zhang M, Kwasny MM, et al. The relationship between clinical, momentary, and sensor-based assessment of depression. Int Conf Pervasive Comput Technol Healthc 2015:2015.

[8] Wahle F, Kowatsch T, Fleisch E, et al. Mobile sensing and support for people with depression: a pilot trial in the wild. JMIR Mhealth Uhealth 2016;4(3):e111.

[9] Burton C, McKinstry B, Szentagotai Tătar A, et al. Activity monitoring in patients with depression: a systematic review. J Affect Disord 2013;145(1):21-8.

[10] Servant D, Logier R, Mouster Y, et al. [Heart rate variability. Applications in psychiatry]. Encéphale 2009;35(5):423-8

[11] Dekker JM, Crow RS, Folsom AR, et al. Low heart rate variability in a 2-minute rhythm strip predicts risk of coronary heart disease and mortality from several causes: the ARIC Study. Atherosclerosis risk in communities. Circulation 2000;102(11):1239-44.

[12] Kemp AH, Quintana DS, Gray MA, et al. Impact of depression and antidepressant treatment on heart rate variability: a review and meta-analysis. Biol Psychiatry 2010;67(11):1067-74.

[13] Jangpangi D, Mondal S, Bandhu R, et al. Alteration of heart rate variability in patients of depression. J Clin Diagn Res 2016;10(12):CM04-6.

[14] Nahshoni E, Aravot D, Aizenberg D, et al. Heart rate variability in patients with major depression. Psychosomatics 2004;45(2):129-34.

[15] Chang HA, Chang CC, Kuo TB, et al. Distinguishing bipolar II depression from unipolar major depressive disorder: differences in heart rate variability. World J Biol Psychiatry 2015;16:351-60.

[16] Agelink MW, Boz C, Ullrich H, et al. Relationship between major depression and heart rate variability. Clinical consequences and implications for antidepressive treatment. Psychiatry Res 2002;113:139-49.

[17] O'Regan C, Kenny RA, Cronin H, et al. Antidepressants strongly influence the relationship between depression and heart rate variability: findings from The Irish Longitudinal Study on Ageing (TILDA). Psychol Med 2015;45(3):623-36.

[18] Lin HP, Lin HY, Lin WL, et al. Effects of stress, depression, and their interaction on heart rate, skin conductance, finger temperature, and respiratory rate: sympathetic-parasympathetic hypothesis of stress and depression. J Clin Psychol 2011;67:1080-91

[19] Ávila Moraes C, Cambras T, Diez-Noguera A, et al. A new chronobiological approach to discriminate between acute and chronic depression using peripheral temperature, rest-activity, and light exposure parameters. BMC Psychiatry 2013;13:77.

[20] Avery DH, Shah SH, Eder DN, et al. Nocturnal sweating and temperature in depression. Acta Psychiatr Scand 1999;100(4):295-301.

[21] Daimon K, Yamada N, Tsujimoto T, et al. Circadian rhythm abnormalities of deep body temperature in depressive disorders. J Affect Disord 1992;26(3):191-8.

[22] Souetre E, Pringuey D, Salvati E, et al. [Circadian rhythms of the central temperature and blood cortisol in endogenous depression]. Encéphale 1985;11(5):185-98 [French]

[23] Abdullah S, Matthews M, Frank E, et al. Automatic detection of social rhythms in bipolar disorder. J Am Med Inform Assoc 2016:23(3):538-43.

[24] Faurholt-Jepsen M, Vinberg M, Christensen EM, et al. Daily electronic selfmonitoring of subjective and objective symptoms in bipolar disorder-the MONARCA trial protocol (MONitoring, treAtment and pRediCtion of bipolAr disorder episodes): a randomised controlled single-blind trial. BMJ Open $2013 ; 3(7)$.

[25] Faurholt-Jepsen M, Vinberg M, Frost M, et al. Daily electronic monitoring of subjective and objective measures of illness activity in bipolar disorder using smartphones-the MONARCA II trial protocol: a randomized controlled singleblind parallel-group trial. BMC Psychiatry 2014;14:309. 
[26] Maxhuni A, Muñoz-Meléndez A, Osmani V, et al. Classification of bipolar disorder episodes based on analysis of voice and motor activity of patients. Pervasive Mob Comput 2016.

[27] Alonso-Martín F, Malfaz M, Sequeira J, et al. A multimodal emotion detection system during human-robot interaction. Sensors (Basel) 2013;13(11):15549-81.

[28] Grünerbl A, Muaremi A, Osmani V, et al. Smartphone-based recognition of states and state changes in bipolar disorder patients. IEEE J Biomed Health Inform 2015;19(1):140-8.

[29] Karam ZN, Mower Provost E, Singh S, et al. Ecologically valid long-term mood monitoring of individuals with bipolar disorder using speech. In: ICASSP, IEEE International Conference on Acoustics, Speech and Signal Processing - Proceedings 2014, p. 4858-62.

[30] https://umclinicalstudies.org/HUM00000606.

[31] Javelot H, Spadazzi A, Weiner L, et al. Telemonitoring with respect to mood disorders and information and communication technologies: overview and presentation of the PSYCHE project. Biomed Res Int 2014;2014:104658.

[32] Mariani S, Migliorini M, Tacchino G, et al. Clinical assessment in bipolar patients by means of HRV features obtained with a sensorized tee-shirt. Conf Proc IEEE Eng Med Biol Soc 2012;2012:2240-50.

[33] Valenza G, Nardelli M, Lanatà A, et al. Wearable monitoring for mood recognition in bipolar disorder based on history dependent long-term heart rate variability analysis. IEEE J Biomed Health Inform 2014;18(5):1625-30.

[34] Faurholt-Jepsen M, Kessing LV, Munkholm K. Heart rate variability in bipolar disorder: a systematic review and meta-analysis. Neurosci Biobehav Rev 2017;73:68-80.

[35] Lee JS, Kim B, Hong Y, et al. Heart rate variability in the subsyndromal depressive phase of bipolar disorder. Psychiatry Clin Neurosci 2012;66(4):361-6.

[36] Faurholt-Jepsen M, Brage S, Kessing LV, et al. State-related differences in heart rate variability in bipolar disorder. J Psychiatr Res 2017;84:169-73.
[37] Chang HA, Chang CC, Tzeng NS, et al. Heart rate variability in unmedicated patients with bipolar disorder in the manic phase. Psychiatry Clin Neurosci 2014;68(9):674-82

[38] Cohen H, Kaplan Z, Kotler M, et al. Impaired heart rate variability in euthymic bipolar patients. Bipolar Disord 2003;5(2):138-43.

[39] Valenza G, Gentili C, Lanatà A, et al. Mood recognition in bipolar patients through the PSYCHE platform: preliminary evaluations and perspectives. Artif Intell Med 2013;57(1):49-58.

[40] Vanello N, Guidi A, Gentili C, et al. Speech analysis for mood state characterization in bipolar patients. In: Proceedings of the Annual International Conference of the IEEE on Engineering in Medicine and Biology Society (EMBC ‘12), p. 2104-7, San Diego, Calif., USA, August-September 2012.

[41] Beiwinkel T, Kindermann S, Maier A, et al. Using smartphones to monitor bipolar disorder symptoms: a pilot study. JMIR Ment Health 2016;3(1):e2.

[42] Gershon A, Ram N, Johnson SL, et al. Daily actigraphy profiles distinguish depressive and interepisode states in bipolar disorder. Clin Psychol Sci 2016;4(4):641-50

[43] Geoffroy PA, Boudebesse C, Bellivier F, et al. Sleep in remitted bipolar disorder: a naturalistic case-control study using actigraphy. J Affect Disord 2014;158:1-7.

[44] Rock P, Goodwin G, Harmer C, et al. Daily rest-activity patterns in the bipolar phenotype: a controlled actigraphy study. Chronobiol Int 2014;31(2):290-6.

[45] Ogien A. Le raisonnement psychiatrique. Librairie des Méridiens Klincksieck et Cie; 1989.

[46] Bourla A, et al. L'acceptabilité des systèmes d'aide à la décision médicale informatisés en psychiatrie : étude quantitative et qualitative sur une population de psychiatres. Conference : $15^{\mathrm{e}}$ congrès de l'Encéphale, à Palais des congrès, 18 janvier 2017, Paris, Volume: 2017.

[47] Borelle C. Montée des outils standardisés et transformation des formes d'autorité professionnelle. Le cas des pédopsychiatres dans le champ de l'autisme. Sociologie 2016;7:153-68. 